\title{
Guía de la OMS / WFME para la acreditación de la formación médica de grado
}

\author{
WHO/WFME Guidelines for Accreditation of Basic Medical Education
}

Organización Mundial de la Salud y Federación Mundial para la Educación Médica.

Ginebra y Copenhague, 2005

\section{PREFACIO}

La necesidad de reformas y mejora de la calidad en educación médica, el notable incremento de Facultades de Medicina en todo el mundo a lo largo de las últimas décadas, muchas de las cuales se han establecido en condiciones cuestionables, así como la salvaguardia de la calidad de los servicios de salud en un mundo cuya globalización va en continuo aumento y con ello la movilidad de los médicos, ha incrementado el reconocimiento de la acreditación como un instrumento que asegura la calidad.

En 2004, la "Colaboración Estratégica OMSWFME para la mejora de la Educación Médica” estableció un grupo de trabajo internacional sobre acreditación. Basándose en los resultados obtenidos por este grupo de trabajo, la "colaboración estratégi$c a$ " ha formulado este conjunto de instrucciones para la acreditación de las instituciones y de los programas de educación médica básica.

Estas guías o instrucciones han sido desarrolladas como un instrumento de ayuda para las autoridades y agencias nacionales que detentan la responsabilidad sobre la calidad de la educación médica con el fin de garantizar el funcionamiento y la transparencia adecuada de los sistemas de acreditación existentes o bien como un instrumento que sirva de ayuda para la puesta en marcha en aquellos países o regiones en los que hasta ahora no existían, nuevos sistemas de acreditación.

Estas guías o instrucciones son fruto de un consenso resultante de las deliberaciones del grupo de trabajo cuya composición incorporaba un amplio abanico de expertos internacionales. Estas guías constituyen una contribución razonable al complejo tema de la acreditación el cual está actualmente influenciando la educación superior en general en todo el mundo. Se espera que estas guías tengan un impacto significativo en la mejora de la calidad de la educación médica. En un futuro, dependiendo de la experiencia acumulada puede llegar a ser necesario hacer una revisión de este documento.

Ginebra / Copenhague, Mayo 2005

\section{PREÁMBULO}

La Organización Mundial de la Salud (OMS) y la Federación Mundial de Educación Médica (WFME) comparten el compromiso de la mejora de la educación médica para la mejora de la asistencia. En consecuencia, en el 2004, la OMS y la WFME acordaron constituir una colaboración estratégica con un plan de trabajo a largo plazo diseñado para tener un impacto decisivo sobre la educación médica. Uno de los elementos decisivos de este plan de trabajo lo constituye la ayuda a las instituciones así como a organizaciones y agencias nacionales o regionales con el fin de desarrollar e implementar programas de reforma o introducir sistemas de reconocimiento y acreditación.

En el 2004 la OMS y la WFME establecieron el Grupo de Trabajo internacional de Acreditación. En el mes de Octubre de 2004, los 26 miembros pertenecientes a 23 países, que abarcaban las seis regio- 
nes de la OMS-WFME, se reunieron en un seminario en Copenhague, Dinamarca, para discutir cómo la OMS y la WFME podían contribuir al establecimiento de un sistema de acreditación sostenible. En dicho seminario se remarcó la necesidad existente de disponer de unas guías o instrucciones y el presente documento está basado en las discusiones de dicho seminario.

Las presentes instrucciones OMS-WFME son realmente "recomendaciones", es decir, guías de cumplimiento no obligado. Como otras organizaciones internacionales tales como la Organización de las Naciones Unidas para la Educación, la Ciencia y la Cultura (UNESCO) y la Organización para la Cooperación y el Desarrollo Económico (OCDE), la OMS y la WFME reconocen que los países confieren un gran valor a la soberanía nacional sobre educación y que las políticas y la regulación de la educación, la educación superior y la educación médica constituyen ámbitos de responsabilidad de los gobiernos nacionales y regionales. El papel de OMSWFME consiste en facilitar la acreditación de la educación médica proveyendo instrucciones indicativas basadas en las mejores prácticas así como en caso de que les sea solicitado, ayudar a los países y las regiones en la puesta en marcha de sistemas de acreditación.

Las instrucciones OMS-WFME son globales pero flexibles. La OMS y la WFME reconocen la existencia de diferencias entre países y regiones en relación con la gobernabilidad de la educación médica, las condiciones y los recursos socioeconómicos, los sistemas de provisión de asistencia sanitaria, etc. Por consiguiente, las instrucciones globales para la acreditación de la educación médica OMS-WFME son flexibles y tienen en cuenta el contexto en que deberán ser utilizadas.

Las instrucciones OMS-WFME y la cooperación internacional. La OMS y la WFME recomiendan firmemente la cooperación internacional en la acreditación de la educación médica tanto en lo que concierne al intercambio de información, incluyendo las experiencias en las mejores prácticas, como en la implementación y gestión de los sistemas de acreditación, incluyendo el intercambio de expertos; también recomiendan el reconocimiento recíproco de sus sistemas de acreditación. Las agencias de acreditación deberían someterse voluntariamente a ser inspeccionadas internacionalmente.

La ayuda de la colaboración OMS-WFME para el establecimiento y desarrollo de los sistemas de acreditación basados en las instrucciones se centrará en principio en los países y regiones necesitados de un sistema de acreditación adecuado. Los países y regiones con una larga tradición en acreditación, que disponen de sistemas de acreditación consolidados y reconocidos, no constituirán una prioridad para las actividades de la colaboración OMS$W F M E$, sin embargo, la experiencia de dichos países y regiones será utilizada para crear los sistemas de acreditación en otros países.

La cooperación internacional es imprescindible para resolver los problemas específicos en el establecimiento de sistemas de acreditación de acuerdo con estas instrucciones, en países con sólo una o dos Facultades de Medicina (que presentan dificultades en relación con la independencia y externalidad de los expertos, las evaluaciones y las decisiones). Esta cooperación puede adoptar formas distintas: afiliación de la única Facultad al sistema de acreditación de un país vecino o creación de sistemas de acreditación supranacionales (regionales o subregionales).

\section{ÁMBITO DE APLICACIÓN DE LAS GUÍAS OMS-WFME}

Las guías para la educación médica básica podrían también ser usadas en la acreditación de la educación médica postgraduada y del desarrollo profesional continuo de los médicos.

Las guías para la acreditación de la educación médica básica son de aplicación a toda la educación médica básica, independientemente del tipo de institución o proveedor (público, privado con o sin ánimo de lucro) e independientemente de la forma de impartir el programa (tradicional o mediante educación a distancia).

Las guías contemplan:

- Los requisitos fundamentales de un sistema de acreditación

- El marco legal

- La estructura organizativa

- Los estándares y criterios

- El proceso de acreditación

- Los principales elementos del proceso de acreditación

- Las decisiones sobre la acreditación

- La publicación de las decisiones

- Los beneficios de la acreditación 


\section{Requisitos fundamentales de un sistema de acreditación}

El requisito básico es que el sistema de acreditación radique en una organización fiable y reconocida por todos los agentes implicados: las facultades de medicina, los estudiantes, la profesión, el sistema de salud y la población. La confianza debe basarse en la competencia académica, la eficiencia y en la equidad del sistema. Estas características del sistema deben ser conocidas por los usuarios y consecuentemente el sistema debe poseer un alto grado de transparencia.

\section{El marco legal}

El sistema de acreditación debe actuar dentro de un marco legal. El sistema debe actuar de acuerdo con cualquier ley o decreto gubernamental que se haya establecido; el instrumento regulador consistirá fundamentalmente en normas y reglas aprobadas por el gobierno. El marco legal debe garantizar la autonomía del sistema de acreditación y asegurar que la evaluación de la calidad que realice sea independiente del gobierno, de las facultades de medicina y de la profesión.

El marco legal debe permitir que los organismos de acreditación establezcan estándares, realicen evaluaciones periódicas y concedan o denieguen la acreditación a las facultades de medicina y a sus programas en educación médica. El marco legal ha de fijar el tamaño y la composición del comité o consejo de acreditación y permitir que este decida de acuerdo con las normas legales, especificando el procedimiento para la acreditación, incluyendo las fechas para las visitas de los comités de evaluación. Además, el marco legal debe incluir normas referentes a la declaración de conflictos de intereses y a los procedimientos para gestionar las quejas o las reclamaciones.

\section{Estructura organizativa}

El órgano o agencia de acreditación debe tener un comité o consejo de acreditación y una unidad administrativa. Para tareas específicas como una evaluación externa, la visita a la institución debe ser fijada por el comité o consejo.

El comité o consejo de acreditación debe contar con un número limitado de miembros (Ej. 9-15 miembros). Los miembros deben ser altamente considerados y respetados en la profesión y preferible- mente de nivel internacional. La mayoría de los miembros deben poseer experiencia educativa en medicina.

Todos los grupos de los principales agentes deben estar representados en el comité de acreditación. Se sugiere que aproximadamente un tercio de los miembros del comité o consejo pertenezcan al "staff" académico, al "staff" de gestión y sean personal "senior" a tiempo completo de las facultades de medicina y nombrados por ellas; aproximadamente otro tercio de los miembros deben provenir de la profesión médica, incluyendo médicos hospitalarios, médicos que trabajen en la comunidad y médicos generalistas y deben ser nombrados por las organizaciones profesionales; finalmente otro tercio de los miembros debe proceder de otros agentes principales, incluyendo autoridades gubernamentales responsables de la educación médica o del sistema de salud, organismos reguladores, estudiantes, otras profesiones sanitarias relacionadas, o la población.

El comité o equipo que realice una visita de evaluación debe estar compuesto por 3-5 miembros, la mayoría de los cuales deben tener experiencia en el campo de la educación médica o de la docencia en medicina. Uno de los miembros debería proceder del área de las ciencias biomédicas básicas y otro de la de las disciplinas clínicas. A ser posible, al menos un miembro debería conocer el país o región y su idioma. Preferiblemente, al menos un miembro debería ser un experto de otro país.

\section{Estándares o criterios}

Los estándares o criterios deben estar predeterminados, consensuados y deben ser públicos. Los criterios a utilizar como base para el proceso de acreditación (para la autoevaluación, la evaluación externa, recomendaciones y la decisión final sobre la acreditación) deben ser los estándares globales de la WFME para la mejora de la calidad de la educación médica básica, con las especificaciones necesarias a nivel nacional y/o regional o un conjunto de estándares comparables.

\section{El proceso de acreditación}

El proceso de acreditación debe incluir los siguientes niveles:

- Una autoevaluación

- Una evaluación externa basada en el informe de autoevaluación y una visita in situ a la institución.

- Un informe final del comité que ha hecho la 
revisión del informe de autoevaluación y la visita después de haber realizado la evaluación externa, y que debe incluir recomendaciones referentes a la decisión de acreditación

- La decisión sobre la acreditación

Las facultades de medicina deberían estar informadas de los miembros que se han propuesto para la revisión del informe de autoevaluación y la visita in situ, y se les debería dar la oportunidad de dirigir la atención a conflictos o intereses potenciales.

El proceso de acreditación también debería permitir a la unidad administrativa de la agencia de acreditación y al comité designado para la revisión y la visita, requerir información suplementaria y aclaraciones sobre el informe de autoevaluación antes de realizar la visita in situ.

Además, se debería proporcionar a la facultad de medicina, el borrador del informe escrito por el comité que haya realizado la evaluación externa o la visita, incluyendo las recomendaciones, con el objetivo de corregir errores de hecho antes de que el informe y las recomendaciones sean presentadas al comité de acreditación.

\section{Elementos principales del proceso de acreditación}

Autoevaluación: El propósito de la autoevaluación es obtener una descripción y análisis de la propia institución así como su programa en relación a los estándares y criterios predeterminados. A parte de ser la base para el proceso de acreditación, la autoevaluación debería considerarse como un instrumento importante de planificación para permitir a la institución conseguir información sobre sus fortalezas y debilidades e identificar áreas para la mejora de la calidad de su programa. La autoevaluación debe ser comprehensiva y cubrir todas las áreas incluidas en los estándares de la WFME:

- misión y objetivos

- programa educativo

- evaluación de los estudiantes

- estudiantes (aspectos distintos de la evaluación, incluyendo la selección, el número, etc.)

- staff académico / profesores

- recursos educativos

- evaluación del programa

- gobernabilidad y administración

- renovación continua

La autoevaluación debe ser precisa y basada en evidencias. Se pueden utilizar todo tipo de materiales o datos, tanto los ya existentes como nuevos.

La institución debe decidir como se debería organizar el trabajo. La elaboración del informe de autoevaluación se podría asignar a un comité ya existente o a un grupo de de trabajo designado. No obstante, se deben implicar a representantes de todas las disciplinas / departamentos, de los diferentes tipos de staff académico y administrativo y de los diferentes tipos de estudiantes.

La agencia de acreditación debería dar soporte a las facultades de medicina elaborando instrucciones referentes a la estructura y contenido del informe de autoevaluación.

Visitas in situ: Los propósitos de las visitas in situ son proporcionar una validación externa de las conclusiones de la autoevaluación referentes al cumplimiento de los estándares, y, si es necesario, adquirir información suplementaria.

Las visitas deben durar de dos a cinco días y como mínimo dos días. La información se recaba durante la visita a través de varios métodos: recolección de documentos y estadísticas (por ejemplo guías de estudio, listas de comprobación y material estadístico sobre la tasa de éxito en los exámenes); entrevistas individuales (con el decano, los jefes de departamento, etc.) y entrevistas en grupo (en reuniones con los comités o grupos responsables de la autoevaluación, comités curriculares, estudiantes, etc.); y por observación directa (durante las visitas a las instalaciones, departamentos y aulas).

La visita in situ debería finalizar con el feedback del comité externo de la revisión o de la visita a la facultad de medicina. Los miembros del comité deberían presentar brevemente sus hallazgos e impresiones preliminares a una audiencia, decidida por la institución y que incluyera a las autoridades académicas.

La agencia de acreditación debería dar soporte a las facultades de medicina elaborando directrices para establecer el programa de la visita in situ.

El Informe final: El comité que realiza la visita debe manifestar claramente en su informe final el cumplimiento o la falta de cumplimiento de los estándares o criterios específicos y debe dar cuenta de la evidencia que dan soporte a las evaluaciones. El informe debe concluir con recomendaciones al comité o consejo de acreditación referentes a la decisión sobre la acreditación.

En raras ocasiones, pueden existir circunstancias específicas de una facultad de medicina que hagan imposible cumplir uno o más estándares. Por ejem- 
plo, la facultad puede no tener jurisdicción sobre la admisión de estudiantes. En estos casos, se debe proporcionar a los evaluadores externos explicaciones adecuadas para entender, y si es el caso, no tomar en consideración estos temas.

\section{Decisiones sobre acreditación}

Las decisiones sobre acreditación deben ser tomadas exclusivamente sobre la base del cumplimiento o incumpliendo de los criterios o estándares. La acreditación debe ser válida para un periodo de tiempo fijado previamente. La duración de la acreditación completa (por ejemplo 5-12 años) debe ser decidida en general.

Categorías de las decisiones sobre acreditación:

- La acreditación completa para el máximo periodo de tiempo debe ser concedida si se cumplen todos los criterios o estándares.

- La acreditación condicional, se entiende como una acreditación concedida para todo el periodo de tiempo establecido pero con unas condiciones que serán revisadas después de un corto periodo para comprobar si se han corregido las deficiencias y cumplido las condiciones impuestas.

La acreditación condicional puede utilizarse en casos donde un pequeño número de criterios o estándares se cumplen parcialmente o en aquellos casos en los que más criterios o estándares no se cumplen. La importancia del problema debe reflejarse en la especificación de las condiciones impuestas.

- Debe denegarse o retirar la acreditación si son demasiados los criterios o estándares que no se cumplen, y que suponen deficiencias importantes en la calidad de los programas y difíciles de ser corregidos en pocos años.

\section{Comunicación pública de las decisiones sobre la acreditación}

Las decisiones sobre la acreditación de los programas médicos deben ser hechas públicas. También debe considerarse la publicación de los informes en los que se basan las decisiones tomadas, o un resumen de estos informes.

\section{Beneficios de la acreditación según la guías de OMS-WFME}

La WHO y la WFME no acreditan facultades de medicina.

Puede proporcionarse información internacional sobre acreditación. La nueva página web de la OMS "Health Academic Institution Database" publicará en la introducción general de cada país su sistema de acreditación y constará el estado de acreditación individual para cada una de las facultades de medicina. 


\section{APÉNDICE}

Miembros del WHO-WFME JOINT

Task Force de Acreditación

Dr. Ghanim Mustafa Alsheikh RA/HRD

World Health Organization (WHO)

Regional Office for the Eastern Mediterranean

Abdul Razzak El Sanhouri Street

Nasr City 11371

Cairo, Egipto

alsheikhg@emro.who.int

Professor Arjuna P. R. Aluwihare, Acting President South East Asian Regional Association for Medical Education (SEARAME)

University of Peradenyia, Medical Faculty

Peradenyia, Sri Lanka

aluwihare@mail.pdn.ac.lk

Dr. James Appleyard, President

The World Medical Association (WMA)

13, ch. du Levant

CIB - Bâtiment A

01210 Ferney-Voltaire, Francia

Jimappleyard2510@aol.com

Dr. Carol A. Aschenbrener

LCME Secretary 2004-05

The Liaison Committee on Medical Education

2450 N Street NW

Washington, DC 20037, USA

caschenbrener@aamc.org

Professor Ibrahim H. Banihani, President,

Association for Medical Education in the

Eastern Mediterranean Region (AMEEMR)

Dean of Faculty of Medicine, Jordan University of Science

\& Technology, P. O. Box 3030,

Irbid-22100, Jordania

banihani@just.edu.jo

omayabanihani@yahoo.com

Professor Abdelmajid Belmahi

President, Conférence Internationale des Doyens et

des Facultés des Médecine

d'Expression Francaise, (CIDMEF)

Dean of Faculty of Medicine of Rabat

Rabat, Marruecos

belmahi@hotmail.com

abelmahi@medramo.ac.ma

Professor Cheng Boji, President

Association for Medical Education in the Western

Pacific Region (AMEWPR)

Office of International Cooperation, Peking

University Health Science Center, 38 Xueyuan

Road Haidian District, Beijing 100083, P.R. China

chengbj@tree.bjmu.edu.cn
Dr. Francisco Campos

Department of Human Resources for Health

WHO Headquarters, Geneva Avenue Appia

1211 Geneva 27, Suiza

camposfr@hotmail.com

Professor, Sir Graeme Catto, President

General Medical Council,

178 Great Portland Street,

London W1W 5JE. RU

opce@gmc-uk.org

graeme.catto@kcl.ac.uk

Professor Leif Christensen, Senior Advisor

World Federation for Medical Education (WFME)

Faculty of Health Sciences, University of Copenhagen

The Panum Institute

Blegdamsvej 3, DK-2200 Copenhagen N, Dinamarca

lec@adm.ku.dk or wfme@wfme.org

Ms. Janet Clevenstine

Department of Human Resources for Health

WHO Headquarters, Geneva Avenue Appia

1211 Geneva 27, Suiza

clevenstinej@who.int

Dr. Kendall Crookston

Fulbright visiting professor

World Federation for Medical Education (WFME)

Faculty of Health Sciences, University of Copenhagen

The Panum Institute

Blegdamsvej 3, DK-2200 Copenhagen N, Dinamarca wfme@wfme.org

Professor Eva Egron-Polak, Secretary General International Association of Universities (IAU) UNESCO House

1, rue Miollis F-75732 Paris Cedex 15, Francia eegron.iau@unesco.org

Professor Michael J. Field

Professor of Medicine, University of Sydney

Associate Dean and Head, Northern Clinical School

Royal North Shore Hospital

St Leonards NSW 2065 Australia

michaelf@medicine.usyd.edu.au

Dr. Akpa Gbary

World Health Organization (WHO)

Regional Office for Africa

Cite du Djoue P.O. Box 6

Brazzaville, Congo

gbarya@afro.who.int

Dr. Charles Godue

Pan American Health Organization

525 Twenty-third Street, N.W.

20037-2895 Washington DC, USA

goduecha@paho.org 
Professor Janet Grant

Director

Open University Centre for Education in Medicine

27 Church Street

Hampton, Middlesex

TW 12 2EB, RU

j.r.grant@open.ac.uk

Dr. P. T. Jayawickramarajah

World Health Organization (WHO)

Regional Office for South-East Asia

World Health House

Indraprastha Estate

Mahatama Gandhi Marg

110002 New Delhi, India

jayawickramarajahp@whosea.org

Dr. Hans Karle, President

World Federation for Medical Education (WFME)

University of Copenhagen, Faculty of Health Sciences

The Panum Institute

Blegdamsvej 3, DK-2200, Copenhagen N. Dinamarca

wfme@wfme.org

Professor Stefan Lindgren

Institutionen för medicin

Universitetssjukhuset MAS

20502 Malmö, Suecia

Stefan.Lindgren@Medforsk.MAS.lu.se

Prof. Thanyani J. Mariba, President

International Association of Medical Regulatory Authorities

Health Professions Council of South Africa

Dean: Faculty of Health Sciences

University of Pretoria PO Box 667

Arcadia, Pretoria, Sudáfrica

Thanyani.Mariba@up.ac.za

Fred Martineau

Medical Student

Moorland

Box End Road

Bromham, Bedford MK43 8LU, RU

fredmart@hotmail.com

Professor Hugo Mercer

Evidence and Information for Policy/HRH/ Human

Resources for Health

World Health Organization

Rue Appia 20

1211-Ginebra 21, Suiza

mercerh@who.int

Dr. Nadia Z. Mikhael

Royal College of Physicians and Surgeons of Canada

774 Echo Drive

Ottawa, Notario, Canadá, K1S 5N8

nmikhael@rcpsc.edu
Dr. Azim Mirzazadeh, Director

Evaluation \& Accreditation, Division of Secretariat

Secretariat of Iranian Council for Graduate

Medical Education (ICGME)

P.O. Box 14155-6476

Teherán, Irán

mirzazad@sina.tums.ac.ir

Professor J.P. deV van Niekerk, President

Association of Medical Schools in Africa (AMSA)

Private Bag X1, Pinelands 7430, Cuidad del Cabo,

Sudáfrica

jpvann@samedical.org

Dr. Ezekiel Nukuro

World Health Organization (WHO)

Regional Office for the Western Pacific

United Nations Avenue, P.O. Box 2932

1000 Manila, Filipinas

nukuroe@wpro.who.int

Dr. Jørgen Nystrup, Senior Advisor, Past President Association for Medical Education in Europe (AMEE) Roskilde Amtssygehus

Smedegade 10-16

4000 Roskilde, Dinamarca

rfjn@ra.dk

Özgür Onur

Director on Medical Education

International Federation of Medical Students'

Associations (IFMSA)

Gringelsbach 61

52080 Aachen, Alemania

lwfme@ifmsa.org

Professor Albert Oriol-Bosch

Fundación Educación Médica y de Ciencias de la Salud

Barcelona, España

aoriolb@terra.es

Professor Mikhail A. Paltsev, Rector

I.M. Sechenov Moscow Medical Academy

Trubetskaia ul. 8, bld.2

119881, Moscú, Rusia

mma-sechenov@mtu-net.ru

Dr. Galina Perfilieva, SCS-HRP

World Health Organization (WHO)

Regional Office for Europe

Scherfigsvej 8

2100 København Ø, Dinamarca

gpe@euro.who.int

Dr. Pablo Pulido, Executive Director

Panamerican Federation of Association of Medical Schools

(PAFAMS), FEPAFEM

Apartado Postal 60411, Caracas, 1080-A, Venezuela

FEPAFEMPAFAMS@CCS.INTERNET.VE 
Professor Peter C. Rubin, Dean,

Faculty of Medicine and Health Sciences

University of Nottingham,

Queen's Medical Centre

Nottingham NG7 2UH, RUK

peter.rubin@nottingham.ac.uk

Professor, Dr. Sharifah H. Shahabudin

Director, Quality Assurance Division

Department of Higher Education

Ministry of Education

Damansara Town Centre

Kuala Lumpur, Malasia

sharifa@pop.jaring.my or drsharifah@moe.gov.my

Professor Emery A. Wilson

Past Chair of LCME

University of Kentucky Medical Center

800 Rose Street

Lexington, KY 40536-0084. USA

ewilson@uky.edu

lmashe2@email.uky.edu
Dr. Ichiro Yoshida

Chairman of International Relations Committee

Japan Society for Medical Education

Professor of Medical Education

Kurume University School of Medicine

67 Asahimachi

Kurume, Japón

iyoshida@med.kurume-u.ac.jp

Dr. phil. nat., Karl Zbinden-Baertschi

Swiss Accreditation Agency

Wissenschaftlicher Mitarbeiter

Organ fuer Akkreditierung und Qualitaetssicherung der Schweizerischen Hochschulen oaq

Effingerstrasse 58

CH-3008 Berna, Suiza

karl.zbinden@oaq.ch 